\title{
Dynamic Simulations of Quantum Cascade Lasers Beyond the Rotating Wave Approximation
}

\author{
Michael Riesch*, Petar Tzenov, and Christian Jirauschek \\ Department of Electrical and Computer Engineering, Technical University of Munich (TUM), Arcisstr. 21, 80333 Munich, \\ Germany
}

\begin{abstract}
We present long-term dynamic simulations of the optical and carrier dynamics in quantum cascade lasers, obtained by numerically solving the Maxwell-Bloch equations without invoking the rotating wave approximation. We discuss the applied numerical methods and parallelization techniques used to obtain the required numerical efficiency and accuracy, and show results for a mode-locked terahertz quantum cascade laser.
\end{abstract}

\section{Introduction}

The quantum cascade laser (QCL) is a type of semiconductor laser that operates in the mid-infrared and terahertz regime. This is achieved by utilizing intersubband transitions between quantized states of a multiple-quantum well active region, rather than interband transitions as in a conventional laser diode. A main application is spectroscopy [1], since the absorption lines of many molecules lie in this frequency regime. By suitable quantum engineering of the active region, not only the spectral gain characteristics, but also the nonlinear optical properties can be custom-tailored. The resulting coherent light-matter interaction has been observed to cause multimode instabilities [2], but has subsequently also been exploited in a targeted manner for the generation of actively mode-locked pulses $[3,4]$ and frequency combs [5, 6]. Ultrashort-pulse trains are used for time-resolved measurements and, due to the associated high peak powers, for applications in nonlinear optics. Frequency combs are spectra consisting of equidistant lines which are widely employed for high-precision spectroscopy and metrology. A detailed understanding of the associated QCL dynamics and targeted design optimization can be achieved based on the Maxwell-Bloch equations. Simulations over many hundred or thousand cavity round trips are required to eliminate transient effects, necessitating an efficient and long-term accurate numerical treatment.

In order to reduce the computational effort, the rotatingwave approximation (RWA) has commonly been invoked $[2,7,8,9,10,11,12]$. A notable exception is the simulation of the seeded QCL dynamics [13], which did not employ the RWA but involved only a few round trips. Notably, the RWA is not applicable to broad spectra, such as arise for few-cycle optical pulses and broadband frequency combs. Recent progress in QCL development has led to such cases. In particular, a QCL design emitting over more than an octave has recently been demonstrated, with comb formation over part of the spectrum [14]. Also, for passive mode-locking, which has not yet been realized in QCLs, the emergence of few-cycle pulses is predicted [10].

We have developed a numerical model, enabling long-term dynamic QCL simulations based on an efficient numerical treatment of the full Maxwell-Bloch equations without RWA. In the following, we present our implementation as open-source software project and discuss a possible use case. In detail, we explain the mathematical model that serves as base for our simulations, along with the numerical methods used to solve the model equations. Then, we present the implementation of the solver and the applied parallelization techniques. Finally, the correctness of the implementation is verified and a first test case is presented.

\section{Mathematical Model}

The QCL dynamics can be described based on a semiclassical model, which is illustrated in Fig. 1. To avoid phenomenological transition and dephasing rates emerging in the Bloch equations, these can be coupled to carrier transport simulations which determine the corresponding rates based on a microscopic evaluation of the scattering processes. As a prerequisite, the Schrödinger-Poisson solver determines the wave functions and energy levels of the discretized states in the active region of the laser. An ensemble Monte Carlo (EMC) [15] or density matrix-EMC [16] approach is used to calculate the scattering-induced transition and dephasing rates between these energy levels due to non-radiative processes [17].

The obtained results are fed into the dynamic model, where they constitute the Hamiltonian $\hat{H}_{0}$, the dipole moment operator $\hat{\mu}$, and the dephasing term $\hat{\rho}_{\text {diss }}$. The coherent lightmatter interaction is modeled by distributing a sufficiently large number of quantum mechanical systems along the propagation direction of the electromagnetic waves. Each system's behavior is governed by a Bloch equation (or more generally Liouville-von Neumann equation). The classical model of the optical field describes the field components 
$E_{z}(x, t)$ and $H_{y}(x, t)$ using Maxwell's equations in one dimension.

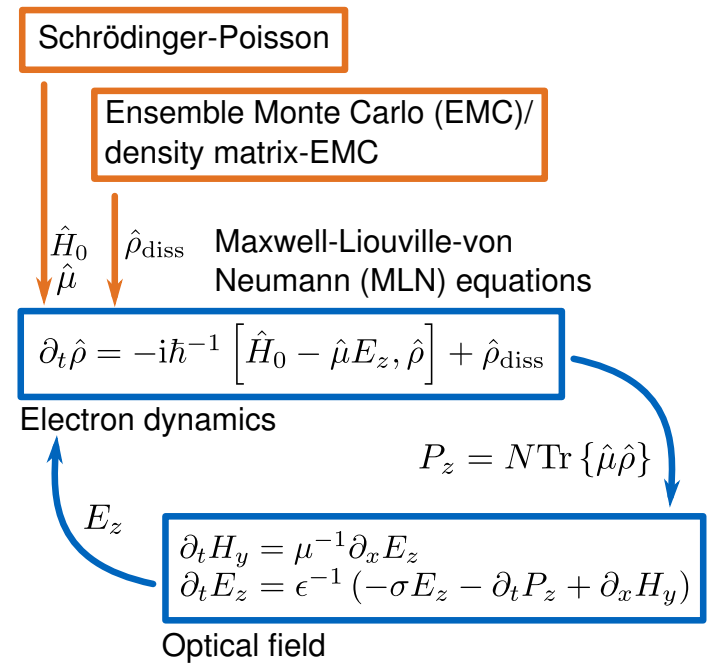

Figure 1. The mathematical model of a quantum cascade laser (QCL) consists of the prerequisite steps (marked in orange) and the Maxwell-Liouville-von Neumann (MLN) equations (blue). The Liouville-von Neumann equation gives the time evolution of the density matrix $\hat{\rho}$, where $\hat{H}_{0}$ is the Hamiltonian, $\hat{\mu}$ is the dipole moment operator, $\hbar$ is the reduced Planck constant, and $\hat{\rho}_{\text {diss }}$ is the dephasing term. Maxwell's equations for the field components $E_{z}(x, t)$ and $H_{y}(x, t)$ consider the conductivity $\sigma$, the permittivity $\varepsilon$, and the permeability $\mu$ of the active region material as well as the polarization term $P_{z}(x, t) . N$ is the density of quantum mechanical particles in the system. Furthermore, $x$ is the propagation direction, $y$ and $z$ are the transversal coordinates, where $z$ denotes the quantum well growth direction, and $t$ is time.

\section{Numerical Methods}

In contrast to many approaches in related literature, the RWA and related slowly-varying amplitude approximation cannot be applied in our simulations. These approximations assume that the optical field is centered around a carrier frequency, which is not the case when considering broadband spectra. Therefore, Maxwell's equations must be directly solved, e.g., using the finite-difference time-domain (FDTD) method [18] or the pseudo-spectral time-domain (PSTD) method [19]. Several methods to couple the Bloch or Liouville-von Neumann equation to Maxwell's equations have been reported. The most prominent approaches are predictor-corrector (PC) methods [20, 21], the operator splitting (OS) technique [22], and variations of the RungeKutta (RK) method (e.g., in [23]). While the search for the ideal numerical method in terms of accuracy and computational efficiency is still in progress, we found that the FDTD-OS method guarantees physically realistic results (such as non-negative level occupations for systems with an arbitrary number of energy levels) and the implementation using the OpenMP standard for parallelization yields reasonable performance $[24,25]$.

\section{Verification and Test Case}

The numerical method was thoroughly validated using test cases from related literature (e.g., the setup from [20]). After the verification, a QCL device consisting of two gain regions that embed an absorber region was simulated, see Fig. 2. This constitutes a colliding-pulse mode-locking setup, where two pulses are expected to form in the cavity. Since thereby the cavity round trip time $T_{R T}$ is effectively reduced, it is expected that such a design is more robust against the short gain recovery times typical for QCLs, which up to now inhibit passive mode-locking [3].

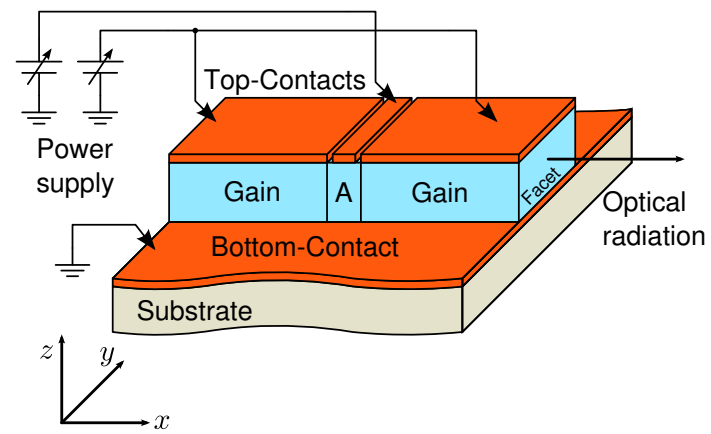

Figure 2. Schematic of the test case setup. The absorber region (A) is embedded between two gain regions. The region types are distinguished by the material and/or the bias voltage.

Indeed, the simulation results (depicted in Fig. 3) demonstrate that this setup produces a pulse train. As anticipated, the time between two pulses is half the round trip time. The corresponding spectrum features peaks separated by twice the round trip frequency and a $10-\mathrm{dB}$ spectral width of $738 \mathrm{GHz}$. Considering that the spectral width is about $20 \%$ of the central frequency, it becomes apparent that the rotating wave approximation would be pushed to its validity limit in this case. As to the performance of the solver, the simulation of 74 round trips took five minutes on a quadcore Intel i7-4790 desktop processor using four threads.

\section{Conclusion and Outlook}

In this contribution we present a highly accurate and efficient solver tool for the Maxwell-Bloch/MLN equations, enabling long-term simulations of the QCL dynamics as required for the modeling of mode-locked and frequency comb operation. It is available as open-source project and will serve as base for future extensions. As an example, we discuss the application of our solver tool to the simulation of the QCL dynamics for a passively mode-locked setup.

As next steps, the MLN equations will be extended to consider more physical effects relevant for the QCL dynamics, such as the implementation of tunneling transport in the Liouville-von Neumann equations and of chromatic dispersion in Maxwell's equations. 


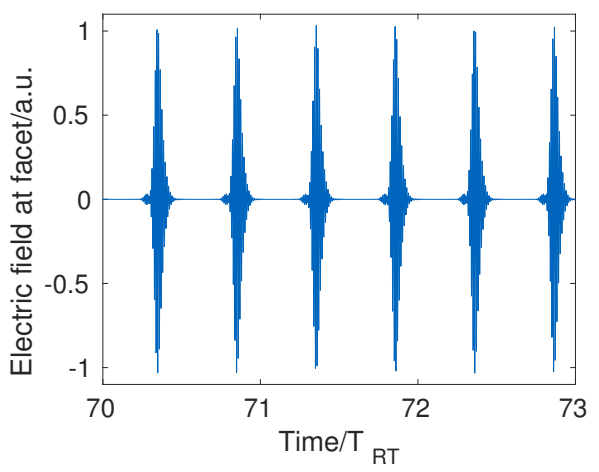

Figure 3. Results of the colliding-pulse mode-locking simulation. The recorded electric field at the left facet of the QCL shows the formation of pulses with an intensity full width at half-maximum duration of $850 \mathrm{fs}$.

\section{Acknowledgments}

This work was supported by the German Research Foundation (DFG) within the Heisenberg program (JI 115/4-2) and under DFG Grant No. JI 115/9-1. The authors gratefully acknowledge the Gauss Centre for Supercomputing e.V. (www.gauss-centre.eu) for funding this project by providing computing time on the GCS Supercomputer SuperMUC at Leibniz Supercomputing Centre (www.lrz.de). Finally, the authors thank Mariem Kthiri and Sebastian Senninger for their help in the development of the mbsolve project.

\section{References}

[1] G. Villares, A. Hugi, S. Blaser, and J. Faist, "Dualcomb spectroscopy based on quantum-cascade-laser frequency combs," Nat. Commun., vol. 5, p. 5192, 2014.

[2] C. Y. Wang, L. Diehl, A. Gordon, C. Jirauschek, F. X. Kärtner, A. Belyanin, D. Bour, S. Corzine, G. Höfler, M. Troccoli, J. Faist, and F. Capasso, "Coherent instabilities in a semiconductor laser with fast gain recovery," Phys. Rev. A, vol. 75, no. 3, p. 031802, 2007.

[3] C. Y. Wang, L. Kuznetsova, V. M. Gkortsas, L. Diehl, F. X. Kärtner, M. A. Belkin, A. Belyanin, X. Li, D. Ham, H. Schneider, P. Grant, C. Y. Song, S. Haffouz, Z. R. Wasilewski, H. C. Liu, and F. Capasso, "Mode-locked pulses from mid-infrared quantum cascade lasers," Opt. Express, vol. 17, no. 15, pp. 12929 12943, 2009.

[4] S. Barbieri, M. Ravaro, P. Gellie, G. Santarelli, C. Manquest, C. Sirtori, S. P. Khanna, E. H. Linfield, and A. G. Davies, "Coherent sampling of active mode-locked terahertz quantum cascade lasers and frequency synthesis," Nat. Photon., vol. 5, no. 5, pp. 306-313, 2011.
[5] A. Hugi, G. Villares, S. Blaser, H. C. Liu, and J. Faist, "Mid-infrared frequency comb based on a quantum cascade laser," Nature, vol. 492, no. 7428, pp. 229233, 2012.

[6] D. Burghoff, T.-Y. Kao, N. Han, C. W. I. Chan, X. Cai, Y. Yang, D. J. Hayton, J.-R. Gao, J. L. Reno, and $\mathrm{Q}$. Hu, "Terahertz laser frequency combs," Nature Photon., vol. 8, no. 6, pp. 462-467, 2014.

[7] V.-M. Gkortsas, C. Wang, L. Kuznetsova, L. Diehl, A. Gordon, C. Jirauschek, M. A. Belkin, A. Belyanin, F. Capasso, and F. X. Kärtner, "Dynamics of actively mode-locked quantum cascade lasers," Opt. Express, vol. 18, no. 13, pp. 13616-13 630, 2010.

[8] C. R. Menyuk and M. A. Talukder, "Self-induced transparency modelocking of quantum cascade lasers," Phys. Rev. Lett., vol. 102, no. 2, p. 023903 , 2009.

[9] A. K. Wójcik, P. Malara, R. Blanchard, T. S. Mansuripur, F. Capasso, and A. Belyanin, "Generation of picosecond pulses and frequency combs in actively mode locked external ring cavity quantum cascade lasers," Appl. Phys. Lett., vol. 103, no. 23, p. $231102,2013$.

[10] M. A. Talukder and C. R. Menyuk, "Quantum coherent saturable absorption for mid-infrared ultra-short pulses," Opt. Express, vol. 22, no. 13, pp. $15608-$ $15617,2014$.

[11] P. Tzenov, D. Burghoff, Q. Hu, and C. Jirauschek, "Time domain modeling of terahertz quantum cascade lasers for frequency comb generation," Opt. Express, vol. 24, no. 20, pp. 23 232-23 247, 2016.

[12] — "Analysis of operating regimes of terahertz quantum cascade laser frequency combs," IEEE Trans. THz Sci. Technol., vol. 7, no. 4, pp. 351-359, 2017.

[13] J. R. Freeman, J. Maysonnave, S. Khanna, E. H. Linfield, A. G. Davies, S. S. Dhillon, and J. Tignon, "Laser-seeding dynamics with few-cycle pulses: Maxwell-Bloch finite-difference time-domain simulations of terahertz quantum cascade lasers," Phys. Rev. $A$, vol. 87, no. 6, p. 063817, 2013.

[14] M. Rösch, G. Scalari, M. Beck, and J. Faist, "Octavespanning semiconductor laser," Nat. Photon., vol. 9, no. 1, pp. 42-47, 2015.

[15] C. Jirauschek and T. Kubis, "Modeling techniques for quantum cascade lasers," Appl. Phys. Rev., vol. 1, no. 1, p. 011307, 2014.

[16] C. Jirauschek, "Density matrix Monte Carlo modeling of quantum cascade lasers," J. Appl. Phys., vol. 122, no. 13, p. 133105, 2017. 
[17] C. Jirauschek and P. Tzenov, "Self-consistent simulations of quantum cascade laser structures for frequency comb generation," Optical and Quantum Electronics, vol. 49, no. 12, p. 414, 2017.

[18] A. Taflove and S. C. Hagness, Computational Electrodynamics: The Finite-Difference Time-Domain Method. Artech House, 2005.

[19] Q. H. Liu, "The PSTD algorithm: A time-domain method requiring only two cells per wavelength," $M i$ crow. Opt. Technol. Lett., vol. 15, no. 3, pp. 158-165, 1997.

[20] R. W. Ziolkowski, J. M. Arnold, and D. M. Gogny, "Ultrafast pulse interactions with two-level atoms," Phys. Rev. A, vol. 52, no. 4, pp. 3082-3094, 1995.

[21] G. Slavcheva, J. M. Arnold, I. Wallace, and R. W. Ziolkowski, "Coupled Maxwell-pseudospin equations for investigation of self-induced transparency effects in a degenerate three-level quantum system in two dimensions: Finite-difference time-domain study," Phys. Rev. A, vol. 66, no. 6, p. 63418, 2002.

[22] B. Bidégaray, "Time discretizations for MaxwellBloch equations," Numer. Methods Partial Differ. Equ., vol. 19, no. 3, pp. 284-300, 2003.

[23] M. Sukharev and A. Nitzan, "Numerical studies of the interaction of an atomic sample with the electromagnetic field in two dimensions," Phys. Rev. A, vol. 84, no. 4, p. 043802, 2011.

[24] M. Riesch and C. Jirauschek, "mbsolve: An opensource solver tool for the Maxwell-Bloch equations," https://github.com/mriesch-tum/mbsolve, 2017.

[25] —, "Numerical method for the MaxwellLiouville-von Neumann equations using efficient matrix exponential computations," https://arxiv.org/abs/1710.09799, 2017. 